\title{
Iron-Loss Computation Using Matlab and Comsol Multiphysics
}

\author{
O. Wallmark, Senior Member, IEEE and K. Bitsi, Student Member, IEEE
}

\begin{abstract}
In this paper, which is tutorial in nature, a description together with associated code samples is presented for predicting iron losses from a two-dimensional, finite-element model implemented using Comsol Multiphysics and Matlab. Two different conventional iron-loss models are presented and compared. The parameters for the adopted loss models are determined for an M250-35A-type steel and sample loss-density distributions are presented for different electric machine geometries.
\end{abstract}

Index Terms-Eddy currents, electric machinery, finite element modeling, hysteresis, iron losses.

\section{INTRODUCTION}

Thanks to its wide range of different physics that can be modeled, it is the opinion of the authors that Comsol Multiphysics $^{1}$ is a commonly used modeling tool in the academic and industrial research sectors. The experience of a common modeling tool using different physics can be an important enabler when multidisciplinary research projects are to be carried out with actors coming from different disciplines. Modeling electric machinery often involves not only electromagnetic modeling approaches but also aspects including heat transfer, fluid dynamics, and solid mechanics must be taken into consideration.

Due to the complex geometry that an electric machine represents, setting up the geometry from a larger set of parameters is conveniently carried out in the form of scripts. In Comsol Multiphysics, these scripts can be written using either Matlab ${ }^{2}$, Java or Visual Basic but in this work, only Matlab scripts will be considered. Working in a scriptbased environment such as Matlab is advantageous thanks to the powerful tools for manipulating and analyzing also large data outputs (such as a time-stepped finite element solution) that is available. However, compared to other available finite-element based software specifically developed for electric machinery modeling, Comsol Multiphysics is still missing common tools for rapid analysis of different aspects, such as the post-processing determination of the resulting losses in the stator and rotor laminations (here termed iron losses) which is not fully developed and incorporated yet.

This work was supported in part by the Swedish Energy Agency and the STandUP for Energy research collaboration initiative.

O. Wallmark and K. Bitsi are with the Division of Electric Power and Energy Systems, KTH Royal Institute of Technology, Stockholm, Sweden (e-mail: \{owa,bitsi\}@kth.se).

${ }^{1}$ Comsol Multiphysics is a registered trademark of COMSOL AB, Stockholm, Sweden.

${ }^{2}$ Matlab is a registered trademark of The Mathworks Inc. Natick, MA.
The accurate prediction of the iron losses is still a challenging topic. Up to now, the majority of the developed ironloss models is based on the Steinmetz equation and the theory of loss separation [1]. These conventional methods can be proven rather inadequate if a purely-sinusoidal field excitation is considered [2]. Instead, a time-domain approach based on these iron-loss models which takes into consideration the dependence of the iron-loss components on the derivative of the arbitrary magnetic flux density, or a frequency-domain model which incorporates the harmonic impact by including a wide spectrum of harmonics in the analysis are proven to achieve better accuracy [2]-[5]. The research is this field is ongoing. Proposed solutions in order to incorporate the presence of local minima (i.e. minor hysteresis loops) in the magnetization curve include the introduction of correction factors considering the magnitudes of the magnetic fluxdensity reversals $\Delta B_{i}$ [6], as well as the consideration of suitable weighting functions $f(|B|)$ [7]. In addition, an iron loss model that incorporates the phenomenon of skin effect and gives acceptable results for higher order harmonics has been introduced in [8]. Other recent advances are presented in [9]-[13].

\section{A. Contribution and Outline of Paper}

This paper, which is tutorial in nature, provides a detailed description, including sample code, for how iron losses can be predicted by importing data from a solved model in Comsol Multiphysics into Matlab. The use of two conventional iron-loss models is demonstrated. The intention is that the explanations given in the paper are so detailed that the code can easily be incorporated in existing Comsol Multiphysics models implemented using Matlab (naturally, the implemented loss model can also be incorporated directly into Comsol Multiphysics). Since the input of the implemented loss models is the $x$ - and $y$-component of the flux density in each mesh element, parts of the code presented can also be used where Matlab is combined with other finite-element based softwares.

The paper is outlined as follows. In Section II, the adopted loss models are described and their parameters are determined for a M250-35A-type steel. As in the first iron-loss model the resulting eddy-current loss factor $k_{e}$ is a function of the flux-density magnitude, a comparison is made with the classical analytical expression used in the second loss model. In Section III, code for implementing the loss models in Matlab is presented with accompanying code included in 
the appendix. Finally, a comparison between the two ironloss models as well as sample loss-density distributions for different electric machines are reported in Section IV, while conclusions are drawn in Section V.

\section{Adopted Loss Models}

The finite element solutions discussed solve for the flux densities in the vertices of the (triangular) mesh. We assume here that the $x$ - and $y$-components of the flux density in the centroid of the triangular element $i$ are available and we denote these $B_{x, i}(t)$ and $B_{y, i}(t)$, respectively. Sample code for how this export procedure from Comsol Multiphysics to Matlab is detailed in Appendix A. In the first iron-loss model utilized in this paper, $B_{x, i}(t)$ is assumed to be periodic (with the assumed period time $T_{\text {per }}$ ), and thus can be described by the Fourier series expansion

$$
B_{x, i}(t)=\sum_{\nu=1}^{\infty}\left|B_{x, i, \nu}\right| \cos \left[\omega_{\nu} t+\arg \left(B_{x, i, \nu}\right)\right]
$$

where $\omega_{\nu}=2 \pi \nu / T_{\text {per }}$ and the dc (constant) component has been neglected since it will not contribute to the iron losses. The (complex) Fourier coefficients $B_{x, i, \nu}$ can be expressed as

$$
B_{x, i, \nu}=\frac{2}{T_{\text {per }}} \int_{0}^{T_{\text {per }}} B_{x, i}(t) e^{-j \omega_{\nu} t} d t
$$

and are in Matlab readily obtained using the command $f \mathrm{ft}$. Similiar to [3], [4], in this iron-loss model, the average loss density in the triangular mesh element $i$ (belonging to the stator or the rotor lamination) is computed as

$$
\begin{aligned}
p_{\text {loss }, i}= & \sum_{\nu=1}^{\nu_{\max }}\left\{k_{\mathrm{e}}\left(\left|B_{x, i, \nu}\right|\right) f_{\nu}^{2}\left|B_{x, i, \nu}\right|^{2}\right. \\
& \left.+k_{\mathrm{e}}\left(\left|B_{y, i, \nu}\right|\right) f_{\nu}^{2}\left|B_{y, i, \nu}\right|^{2}\right\} \\
& +\sum_{\nu=1}^{\nu_{\max }}\left\{k_{\mathrm{h}}\left(\left|B_{x, i, \nu}\right|\right) f_{\nu}\left|B_{x, i, \nu}\right|^{2}\right. \\
& \left.+k_{\mathrm{h}}\left(\left|B_{y, i, \nu}\right|\right) f_{\nu}\left|B_{y, i, \nu}\right|^{2}\right\} .
\end{aligned}
$$

The total (average) losses in the electrical steel can now be computed as

$$
P_{\text {spec }}=L_{a} \sum_{i} p_{\text {loss }, i} \Delta_{i}
$$

where $\Delta_{i}$ denotes the area of mesh element $i, L_{a}$ is the active length, and the summation should be done over all triangular elements $i$ that belong to the electrical steel.

Fig. 1 depicts loss data of the M250-35A steel obtained from [14] and the respective fitted loss model (3). In order to achieve a good agreement between the data and the model, the loss coefficients $k_{e}$ and $k_{h}$ are derived as functions of the magnetic flux density within the given frequency range (see Fig. 2). The classical expression for $k_{e}$ is

$$
k_{e}=\frac{\pi^{2} \sigma l_{\mathrm{lam}}^{2}}{6}
$$

where $\sigma$ is the electrical conductivity and $l_{\text {lam }}$ the thickness of the lamination, respectively. As it can be seen in Fig. 2 (a), the deviation of $k_{e}$ from the corresponding classical expression is substantial. Thus, the use of core loss coefficients variable with induction is employed in order to mitigate the inaccuracies of the conventional core loss models with constant coefficients [15], [16].

(a)

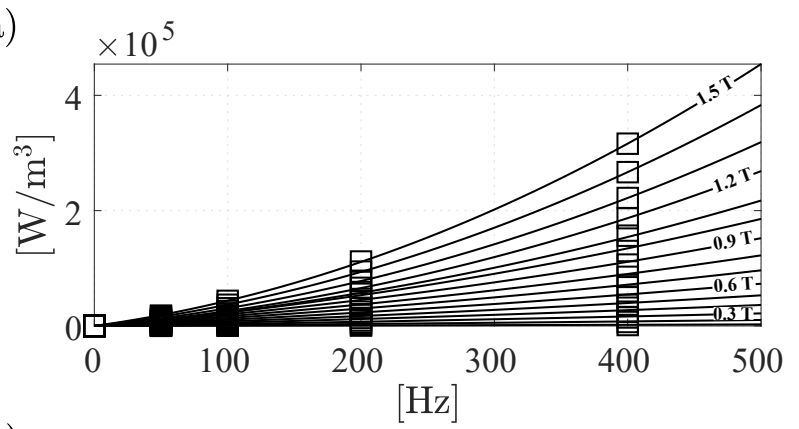

(b)

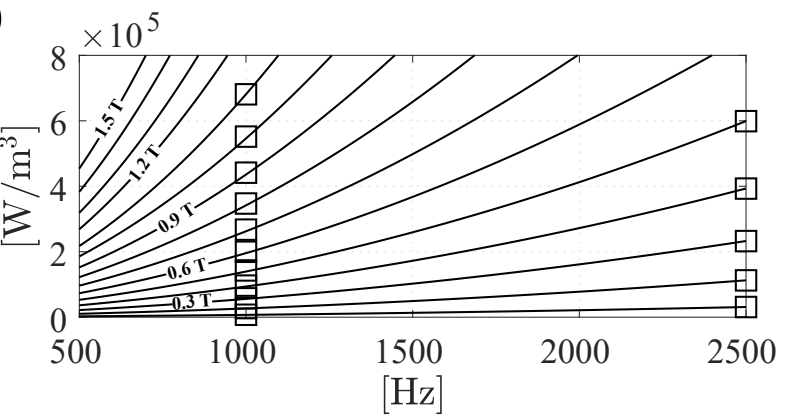

Fig. 1. M250-35A loss data. Squares ( $\square$ ) denote data from [14], assuming a steel density of $7600 \mathrm{~kg} / \mathrm{m}^{3}$, whereas solid lines are derived based on the fitted loss model (3).

The second method that is used in this paper is based on the time-domain three-term iron loss model introduced in [5]. According to this model, the total (average) iron losses in the electrical steel are calculated as

$$
\begin{aligned}
P_{\text {spec }} & =\frac{k_{e}}{2 \pi^{2} T_{\text {per }}} \int_{0}^{T_{\text {per }}}\left\{\left(\frac{d B_{x}}{d t}\right)^{2}+\left(\frac{d B_{y}}{d t}\right)^{2}\right\} d t \\
& +\frac{1}{T_{\text {per }}} \int_{0}^{T_{\text {per }}}\left(\left|H_{x} \frac{d B_{x}}{d t}\right|+\left|H_{y} \frac{d B_{y}}{d t}\right|\right) d t \\
& +\frac{k_{\text {exc }}}{C_{\text {exc }} T_{\text {per }}} \int_{0}^{T_{\text {per }}}\left\{\left(\frac{d B_{x}}{d t}\right)^{2}+\left(\frac{d B_{y}}{d t}\right)^{2}\right\}^{0.75} d t
\end{aligned}
$$

where $k_{e}$ is the classical eddy-current coefficient (5), $k_{\mathrm{exc}}$ the excess-loss coefficient determined through curve fitting on the manufacturer's loss data and $C_{\text {exc }}=8.763363$ a constant derived from the numerical integration of the excess-loss term [5].

\section{MODELS IMPLEMENTATION}

For the implementation of the iron-loss model (3), the magnitudes BXFFT (and, similarly, BYFFT) and the corresponding frequency vector $f$ can be obtained as 
(a)

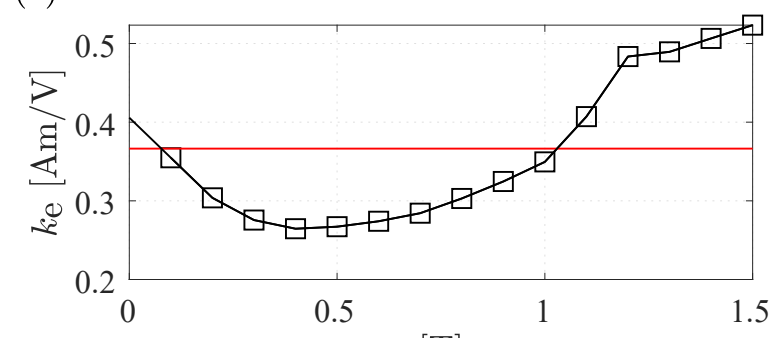

(b)

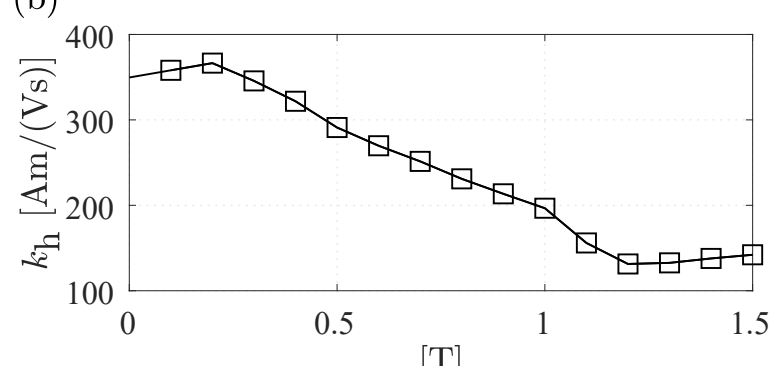

$[\mathrm{T}]$

Fig. 2. Obtained loss coefficients $k_{e}$ and $k_{h}$ as function of flux-density magnitude within the given frequency range shown in Fig. 1. Solid red line in (a) corresponds to $k_{e}$ given by (5).

$\mathrm{BXFFT}=2 . * \mathrm{abs}($ fft $(\mathrm{BX}) . /$ length $(\mathrm{t}))$;

$\operatorname{BXFFT}(1,:)=0.5 . * \operatorname{BXFFT}(1,:)$;

$\mathrm{f}=(0: 1:$ length $(t)-1) . /(t($ end $)-t(1))$;

where BX (and similarly BY) is obtained as outlined in Appendix $A$ and $t$ is the simulation time in which the variation of the flux-density distribution is assumed to correspond to an integer number of periods. Comparing with (3), BXFFT corresponds to $\left|B_{x, i, \nu}\right|$ and $\mathrm{f}$ corresponds to $f_{\nu}$. The loss coefficients keBX and khBX (as functions of the flux density magnitude) based on the loss data BData, keData, and khData (shown using squares ( $\square$ ) in Fig. 2) up to the frequency fMax (corresponding to $\nu_{\max }$ in (3)) can now be obtained as

fIndex=find $(\mathrm{f}<=\mathrm{fMax})$;

keBX=interp1 (BData, keData, BXFFT (fIndex, :),

'linear',' extrap');

khBX=interp1 (BData, khData, BXFFT (fIndex, : ),

'linear',' extrap')';

The (average) iron loss distribution pLoss (in $\left[\mathrm{W} / \mathrm{m}^{3}\right]$ ) can now be calculated as

pLoss =sum ( (fVectMatrixForm. ^2) . *keBX

.* (BXFFT (fIndex, : ) .^2) ) +sum( (fVectMatrixForm) . *keBY

.* (BYFFT (fIndex, :) .^2) ) +sum( (fVectMatrixForm) . *khBX

.* (BXFFT (fIndex, : ).^2) ) +sum ( (fVectMatrixForm) . *khBY

.*(BYFFT (fIndex, : ).^2));

If the iron-loss model (6) is considered instead, the magnitudes HX and HY should be determined using a similar method as in the case of BX and BY (see Appendix A). The (average) iron loss distribution pLoss (in $\left[\mathrm{W} / \mathrm{m}^{3}\right]$ ) can now be obtained as

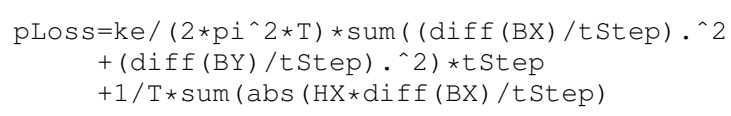

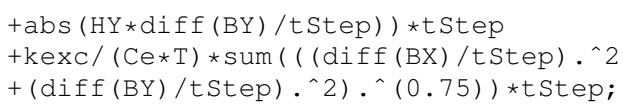

In Appendix B, code for how to import the area of each mesh element meshElArea into Matlab is provided. The total (average) iron losses PLoss (in [W]) using either of the two models can be obtained as

PLoss $=$ La . * pLoss $*$ meshElArea;

\section{SAmple Loss-Density Distributions}

Code for how to obtain the coordinates for each node $\mathrm{XN}$ and YN can be found in Appendix C. Using the patch command, the (average) loss-density distribution can be visualized as

patch $\left(\mathrm{XN}^{\prime}, \mathrm{YN}^{\prime}, \mathrm{pLOSS^{ \prime }}\right)$;

Fig. 3 shows resulting sample loss-density distributions using the iron-loss model (3) for two permanent-magnet and two induction machines, all operating at constant speed and fed with sinusoidal phase currents. Fig. 3a depicts a 4-pole, 24 slot surface-mounted permanent-magnet (SMPM) synchronous machine with a distributed winding. The harmonic content due to the distributed winding is very low and the resulting losses in the rotor lamination is practically negligible. Fig. $3 b$ depicts an 8-pole, 12 slot interior permanent-magnet (IPM) synchronous machine with a double-layer concentrated winding. The harmonic content due to this winding arrangement is substantial which results in relatively large losses also in the rotor laminations. Fig. $3 \mathrm{c}$ and d depict a 2-pole, 36 slot induction machine (IM) with a rotor where the rotor bars are optimized for grid and inverter (variable frequency) operation, respectively. In this case, the largest share of the iron losses are concentrated in the region close to the air gap.

\section{A. A Comparison of the Adopted Iron-Loss Models}

The two adopted iron-loss models are compared for the SMPM synchronous machine and the induction machine shown in Fig. 3(a) and Fig. 3(d), respectively. The results are tabulated in Table I. It can be observed that the ironloss estimations of both loss models follow similar trends for the stator and rotor laminations. In general, a reasonable agreement between the two iron-loss models is ascertained.

TABLE I

COMPARISON OF TOTAL ESTIMATED IRON LOSSES USING THE TWO ADOPTED IRON-LOSS MODELS FOR DIFFERENT MACHINE GEOMETRIES

\begin{tabular}{c|cc|cc} 
& \multicolumn{2}{|c|}{ SMPM (Fig. 3a) } & \multicolumn{2}{c}{ IM (Fig. 3d) } \\
& $P_{\text {stator }}[\mathrm{W}]$ & $P_{\text {rotor }}[\mathrm{W}]$ & $P_{\text {stator }}[\mathrm{W}]$ & $P_{\text {rotor }}[\mathrm{W}]$ \\
\hline $\begin{array}{c}\text { Iron-Loss } \\
\text { Model (3) }\end{array}$ & 151.9 & 0.1 & 117.1 & 17.4 \\
\hline $\begin{array}{c}\text { Iron-Loss } \\
\text { Model (6) }\end{array}$ & 157.2 & 1.5 & 124.6 & 20.6 \\
\hline
\end{tabular}




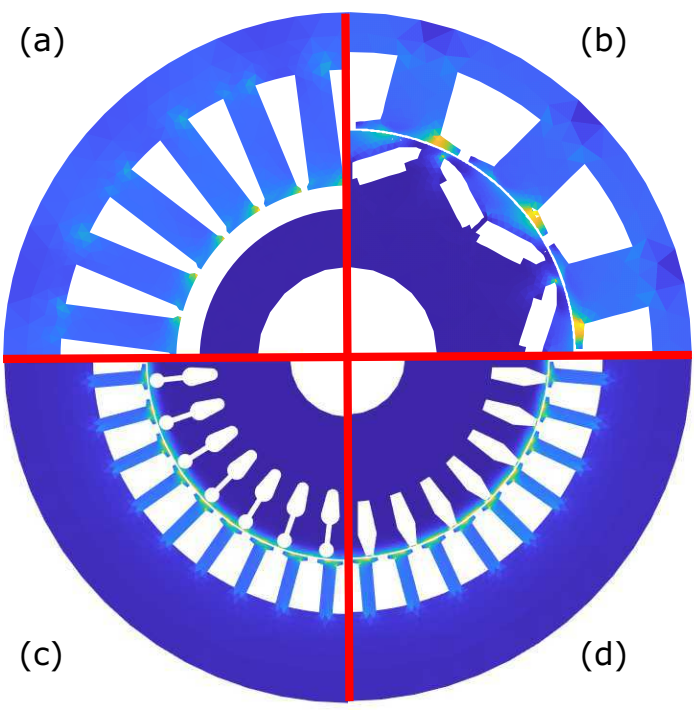

Fig. 3. Resulting loss-density distributions: (a) Four-pole permanent-magnet synchronous machine with surface-mounted magnets distributed winding; (b) Eight-pole permanent-magnet machine with interior-mounted magnets and concentrated winding; (c) Two-pole induction machine with rotor bars shaped optimized for grid-connected start; (d) Two-pole induction machine with rotor bars suited for inverter (variable frequency) operation.

\section{B. A Comparison with Other Software}

In order to compare the outcome of this analysis with results from different software, the IPM machine shown in Fig. $3 \mathrm{~b}$ is considered. The depicted loss-density distribution corresponds to the operating point of $4000 \mathrm{rpm}$ and $85 \mathrm{Nm}$. In [17], the iron losses are determined at the same operating point using the software JMAG and its in-built iron-loss model. The reported results are $509 \mathrm{~W}$ and $90 \mathrm{~W}$ for the stator and rotor, respectively (see page 36 in [17]). Using the first approach described in this paper (3), the corresponding iron losses are $397 \mathrm{~W}$ and $67 \mathrm{~W}$, respectively, and thus are in relative good agreement with the losses predicted in [17]. Reasons for the deviation between the results include different meshes, different parameterizations of the adopted loss model and differently assumed remanent flux density of the permanent magnets. Since the remanent flux density in the permanent magnet material decreases with temperature, for the same torque, a higher current amplitude is required at elevated temperatures which results in a different flux-density distribution and, hence, different iron losses.

\section{CONCLUSiON}

This paper presents sample codes for how the ironloss density distribution can be obtained in a post-process procedure when using Matlab and Comsol Multiphysics. The paper is tutorial in nature and its purpose is to provide a detailed description for how existing, two-dimensional models of electric machinery can be extended to allow for the computation of iron losses. Two conventional iron-loss models are adopted in this paper. In the first model, a Fourier series expansion of the magnetic flux density is deployed for the calculation of iron losses in a spectrum of frequencies, while the loss coefficients are considered to be dependent on the flux-density magnitude. To exemplify this approach, sample loss distributions for two permanent-magnet and two induction machines are demonstrated. The second model is a time-domain three-term model based on the theory of loss separation, where time derivatives of the magneticflux density are utilized for the iron-loss computation. The comparison of these two models shows a good agreement between the iron-loss estimation techniques. The work could easily be modified to incorporate other types of loss model approaches.

\section{APPENDIX}

\section{A. Exporting Flux-Density Data to Matlab}

Firstly it is assumed that a model for predicting flux-density distribution in the electric machine as function of time is solved. With this solution in memory, a duplicate set dSetCopy of the solution is created. In Comsol, every model is divided into selections of different parts of the geometry. For estimating the stator iron losses, only the selection corresponding to the stator lamination is assigned to dSetCopy. This is done by executing the following code:

model.result. dataset.duplicate ('dSetCopy',' dset1') ; model.result.dataset ('dSetCopy'). selection .geom ('geom1', 2);

model.result.dataset ('dSetCopy'). selection . named ('selstatorLam');

Now, the data (time series) of the flux density variation in the centroid of each mesh element belonging to the stator lamination ( $x$ - and $y$-components in separate files) is stored as BX.dat and BY.dat and loaded into Matlab. Including only $\mathrm{BX}$, this can be done with the following code:

model.result. export. create ('BX', 'Data');

model.result. export ('BX'). set ('data',' dSetCopy') ; model.result. export ('BX'). set ('expr', \{'rmm.BX' $\})$; model.result.export ('BX'). set ('pattern','gauss') ; model.result. export ('BX'). set ('unit', $\left.\left\{{ }^{\prime} \mathrm{T}^{\prime}\right\}\right)$;

model.result.export ('BX'). set ('filename', 'BX.dat')) i model.result. export ('BX').run;

dataload ('BX.dat');

$\mathrm{XC}=\operatorname{data}(:, 1)$;

$\mathrm{YC}=\operatorname{data}(:, 2)$;

$\mathrm{BX}=\operatorname{dat} \mathrm{a}(:, 3:$ end $)$;

\section{B. Determining Area Per Mesh Element}

To store the area for triangular mesh element belonging to the stator lamination and load this data into Matlab, the code below can be used.

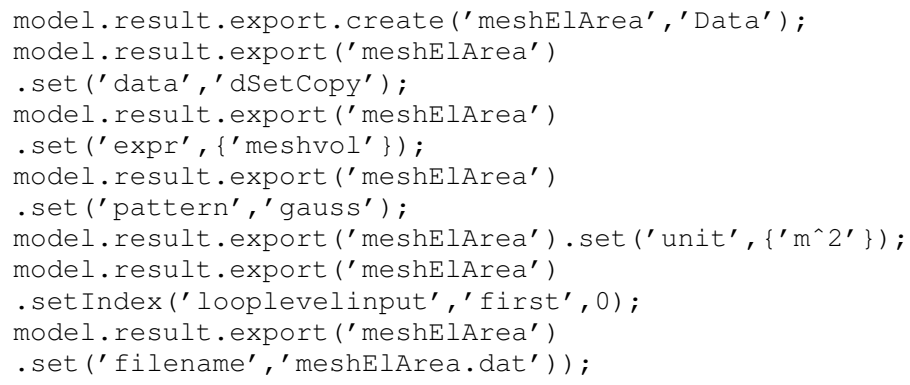


model.result. export ('meshElArea').run;

dataload ('meashArea.dat') ;

meshElArea=data $(:, 3)$;

\section{Determining the Coordinates of Each Node}

The $x$ - and $y$-coordinates (named XN and YN) of each mesh node can be imported into Matlab using the following code:

model.result.export ('meshElArea')

. set ('pattern',' lagrange');

model.result.export ('meshElArea')

. set ('filename','BXN.dat')) ;

model.result.export ('meshElArea')

. setIndex (' looplevelinput', 'first',0);

model.result.export ('meshelArea').run;

dataload ('BXN.dat') ;

$\mathrm{XN}=\operatorname{data}(:, 1)$;

$\mathrm{YN}=\operatorname{data}(:, 2)$;

Now, to identify which set of three-node coordinates corresponds to the coordinates of the centroid of a mesh element (given by $\mathrm{XC}$ and $\mathrm{YC}$ ), the finding-nearest-neighbors command knnsearch is used as

[index $]=k n n s e a r c h\left([X N\right.$ YN $\left.],[X C Y C], ' \mathrm{~K}^{\prime}, 3\right)$; $\mathrm{XN}=\mathrm{XN}($ index $) ; \mathrm{YN}=\mathrm{YN}($ index $)$;

\section{REFERENCES}

[1] A. Krings and J. Soulard, "Overview and comparison of iron loss models for electrical machines," Journal of Electrical Engineering, vol. 10, pp. 162-169, 052010.

[2] D. Kowal, P. Sergeant, L. Dupré, and L. Vandenbossche, "Comparison of iron loss models for electrical machines with different frequency domain and time domain methods for excess loss prediction," Magnetics, IEEE Transactions on, vol. 51, pp. 1-10, 012015.

[3] P. A. Hargreaves, B. C. Mecrow, and R. Hall, "Calculation of iron loss in electrical generators using finite-element analysis," IEEE Transactions on Industry Applications, vol. 48, no. 5, Sept./Oct. 2012.

[4] M. Fratila, A. Benabou, A. Tounzi, and M. Dessoude, "Iron loss calculation in a synchronous generator using finite element analysis," IEEE Transactions on Energy Conversion, vol. 32, no. 2, June 2017.

[5] D. Lin, P. Zhou, W. N. Fu, Z. Badics, and Z. J. Cendes, "A dynamic core loss model for soft ferromagnetic and power ferrite materials in transient finite element analysis," IEEE Transactions on Magnetics, vol. 40, no. 2, pp. 1318-1321, March 2004.

[6] J. Lavers, P. Biringer, and H. Hollitscher, "A simple method of estimating the minor loop hysteresis loss in thin laminations," IEEE Transactions on Magnetics, vol. 14, no. 5, pp. 386-388, Sep. 1978.

[7] A. Arkkio and A. Niemenmaa, "Estimation of losses in cage induction motors using finite-element techniques," in 1992 ICEM, 1992, p. 317-321.

[8] K. Yamazaki and N. Fukushima, "Iron loss model for rotating machines using direct eddy current analysis in electrical steel sheets," IEEE Transactions on Energy Conversion, vol. 25, no. 3, pp. 633-641, Sep. 2010.
[9] K. Yamazaki and Y. Fukushima, "Torque and loss calculation of rotating machines considering laminated cores using post 1-D analysis,' IEEE Transactions on Magnetics, vol. 47, no. 5, pp. 994-997, May 2011.

[10] G. Bramerdorfer and D. Andessner, "Accurate and easy-to-obtain iron loss model for electric machine design," IEEE Transactions on Industrial Electronics, vol. 64, no. 3, Mar. 2017.

[11] M. Petrun and S. Steentjes, "Iron-loss and magnetization dynamics in non-oriented electrical steel: 1-D excitations up to high frequencies," IEEE Access, vol. 8, pp. 4568-4593, 2020.

[12] J. Saitz, "Computation of the core loss in an induction motor using the vector Preisach hysteresis model incorporated in finite element analysis," IEEE Transactions on Magnetics, vol. 36, no. 4, pp. 769773, July 2000

[13] L. Chang, T. M. Jahns, and R. Blissenbach, "Generalized dynamic hysteresis model for improved iron loss estimation of complex flux waveforms," IEEE Transactions on Magnetics, vol. 55, no. 7, July 2019.

[14] Non-oriented electrical steel - Typical data. Surahammars Bruks AB. Accessed Jan. 2nd, 2014. [Online]. Available: http://www.sura.se

[15] M. Popescu and D. M. Ionel, "A best-fit model of power losses in cold rolled-motor lamination steel operating in a wide range of frequency and magnetization," IEEE Transactions on Magnetics, vol. 43, no. 4, pp. 1753-1756, 2007.

[16] A. Boglietti, A. Cavagnino, D. M. Ionel, M. Popescu, D. A. Staton, and S. Vaschetto, "A general model to predict the iron losses in inverter fed induction motors," in 2009 IEEE Energy Conversion Congress and Exposition, 2009, pp. 1067-1074.

[17] H. Zhang, "On eletric machinery for integrated motor drives in automotive applications," Ph.D. Dissertation, KTH Royal Institute of Technology, Stockholm, Sweden, June 2017.

\section{BIOGRAPHIES}

Oskar Wallmark was born in 1976. He received the M.Sc. degree in Engineering Physics and the Ph.D. degree in Electric Power Engineering from the Chalmers University of Technology, Göteborg, Sweden, in 2001 and 2006, respectively, and the Docent degree from the KTH Royal Institute of Technology, Stockholm, Sweden. He is currently an Associate Professor with the Division of Electric Power and Energy Systems, KTH Royal Institute of Technology, Stockholm, Sweden. His current research interests include control and analysis of electric drives, especially for automotive applications.

Konstantina Bitsi was born in Athens, Greece in 1992. She received the M.Sc. degree in Electrical and Computer Engineering from the National Technical University of Athens, Greece, in 2016. She is currently pursuing the Ph.D. degree at the Division of Electric Power and Energy Systems, KTH Royal Institute of Technology, Stockholm, Sweden. Her current research interests include electrical machine design and optimization for automotive applications. 